\title{
Action Patterns in Business Process Models
}

\author{
Sergey Smirnov ${ }^{1}$, Matthias Weidlich ${ }^{1}$, Jan Mendling $^{2}$, and Mathias Weske ${ }^{1}$ \\ 1 Hasso Plattner Institute, Potsdam, Germany \\ \{sergey.smirnov, matthias. weidlich, mathias. weske\}@hpi.uni-potsdam.de \\ 2 Humboldt-Universität zu Berlin, Germany \\ jan.mendling@wiwi.hu-berlin.de
}

\begin{abstract}
Business process management experiences a large uptake by the industry, and process models play an important role in the analysis and improvement of processes. While an increasing number of staff becomes involved in actual modeling practice, it is crucial to assure model quality and homogeneity along with providing suitable aids for creating models. In this paper we consider the problem of offering recommendations to the user during the act of modeling. Our key contribution is a concept for defining and identifying action patterns - chunks of actions often appearing together in business processes. In particular, we specify action patterns and demonstrate how they can be identified from existing process model repositories using association rule mining techniques. Action patterns can then be used to suggest additional actions for a process model. Our approach is challenged by applying it to the collection of process models from the SAP Reference Model.
\end{abstract}

\section{Introduction}

Business process management experiences a large uptake by the industry, as more and more companies analyze and improve their processes to stay competitive. Process models, being formal representations of business processes, facilitate many tasks in the domain of business process management. Thereby, instead of being an art of a few specialists, process modeling becomes a daily routine of office staff. This development implies several challenges in terms of an efficient and effective modeling support. In particular, many staff members have low modeling competence and model only on an irregular basis [20. For this reason, process modeling tools have to incorporate techniques to help these casual modelers to conduct their work in a productive way.

Business process modeling research has revealed several approaches to make modeling more efficient. This research can be classified into two main categories. On the one hand, reference modeling aims to increase productivity based on the reuse principle: models are created for a specific domain and are meant to be customized in different application projects. On the other hand, different types of patterns describe recurring situations in a domain independent way. The potential of both approaches is hardly reflected by current tool features. Whilst most of the pattern sets for processes and workflows are mainly used for

L. Baresi, C.-H. Chi, and J. Suzuki (Eds.): ICSOC-ServiceWave 2009, LNCS 5900, pp. 115-129, 2009.

(C) Springer-Verlag Berlin Heidelberg 2009 
model verification and modeling language analysis, the existing reference models are tightly coupled with their partial domain and can hardly be used in other settings. Against this background, we define a concept of action patterns. In contrast to well known workflow patterns, action patterns are closely related to semantic content of a process model. Meanwhile, unlike reference models, action patterns are abstract enough to be applicable in various domains. In this context, the term action essentially refers to the verb that describes the work content of a textual activity label.

The contribution of this paper is a formal description of action patterns and an approach for identification of patterns in existing process model collections based on association rules mining. The mined action patterns can be used to suggest additional activities to the modeler during a modeling act. We specify two classes of patterns. Co-occurrence action patterns signify sets of actions that are likely to appear jointly in a model. Behavioral action patterns describe how co-occurring actions are related to each other in terms of behavioral constraints. This information allows us to identify the control flow position where an activity has to be added.

The rest of the paper is structured as follows. Section 2 provides a motivating example to illustrate our approach. Section 3 formalizes the action pattern concept and presents two classes of action patterns: co-occurrence action patterns and behavioral action patterns. Section 4 describes the evaluation of our approach by deriving action patterns from the SAP Reference Model. In Section 5 we present an outlook of the related work. Section 6 concludes the paper.

\section{Motivating Example}

An intrinsic complexity of business processes together with process models heterogeneity, originating from a variety of stakeholders and modeling purposes, calls for sophisticated support for process modeling. We distinguish two important drivers for such modeling support. On the one hand, the support aims at facilitating the design of a standalone process model. This kind of modeling support includes means to accelerate process model creation, assure correct model execution semantics, and increase model conciseness. However, the focus is purely on the isolated creation of a dedicated model: the application domain of this model is not taken into account. On the other hand, the rationale behind modeling support might be homogeneity of the modeling efforts. Process models created within a certain domain, might it be an organizational unit or a process model collection, should be modeled in a consistent and similar manner. In this case the emphasis is on avoiding redundancies and contradictions, as well as on enforcing modeling guidelines.

We illustrate the use case of domain-aware modeling support by means of the example in Fig. 11 which shows fragments of two EPCs from the SAP Reference Model [9]. Both business processes originate from the SAP material management and describe production planning. We see that the processes have a similar structure and semantics. For the long-term planning (Fig. 1(a), as well as for the 


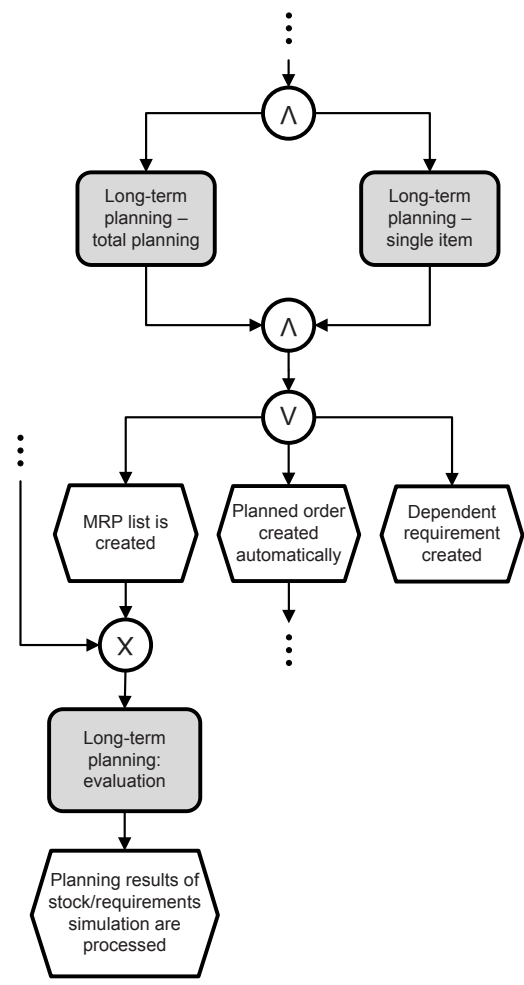

(a) Long-term planning

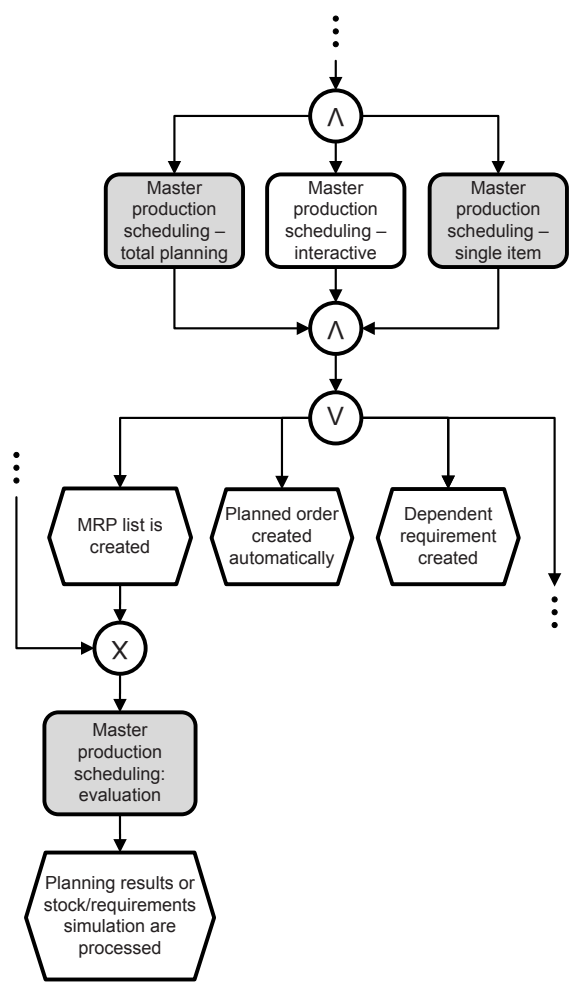

(b) Master production scheduling

Fig. 1. Fragments of two similar planning processes from the SAP Reference Model

master production scheduling (Fig. 1(b)], two similar planning steps are performed concurrently, and in both cases are succeeded by an evaluation. In Fig. 1 we highlight the activities which are interesting for us with grey color. Given these models, the creation of a model for a related process, e.g., a short-time production planning, might be supported as follows. After the modeler creates a function Short-Term Planning - Total Planning, we suggest to insert a concurrent function Short-Term Planning - Single Item on the fly. This recommendation can be derived from the analysis of the already existing models. We might also alert the modeler if he saves the model for short-term planning without having inserted a function for planning evaluation. The modeler might reconsider the modeling decisions and insert such a function, or rename an existing function, which has been intended to model the evaluation step, but was labeled differently (e.g., function planning calculation can be renamed to planning evaluation).

For obvious reasons, such domain-aware modeling support has to take into account semantics of existing process models. In this case semantics is not restricted to the model execution semantics. Instead, semantics has to be given in terms of concepts of the application domain. Applied to Fig. 1, a dependency 
between the planning steps (total planning vs. single-item), as well as their relation to the planning evaluation, are examples of semantic dependencies specific for an application domain. The question how to derive a formalization of domain knowledge is crucial for a domain-aware modeling support.

To formalize domain knowledge, one might apply semantic annotations for all elements of the process. In this case, semantic information is represented in a structural way. This enables straight-forward processing and simplifies the usage for modeling support. However, this approach has an obvious drawback, since it requires semantic annotation of all model elements as a preliminary step. For a large collection of process models such a preliminary step requires enormous efforts and, thus, might not be feasible.

Therefore, we follow another approach relying on the analysis of model element labels. The goal of this analysis is to understand the meaning of labels and extract domain knowledge out of an existing process model collection. Such an approach has to deal with the high ambiguity of a natural language. However, our experience (see [19]), as well as the experience of other researchers (see [4]), proves that label analysis is feasible. Besides, to simplify model comprehension for humans, modelers often stick to one schema when labeling model elements. An example is verb + noun schema employed for activity labeling. Analysis of such labels can be seen as an automated operation. The analysis outcome is the mapping of each activity to an action for which the activity stays in the model. For instance, from an activity labeled with send notification one can derive that action send is performed on object notification.

In this paper we focus on supporting the modeler with recommendations on actions potentially missing in a process model. While such recommendations depend on various model elements and other factors in the general case, we focus on the analysis of activities. There are two major drivers for our work. First, giving recommendations on the missing actions (i.e., activities on the model level) requires exhaustive investigation of the existing ones. Second, a lion's share of business process model semantics is given by the activities. Hence, we formulate the recommendations based on the analysis of activities. To formalize the knowledge extracted from a process model collection we propose to use the notion of action patterns - groups of actions which often appear together in business processes. In the next section we elaborate on the concept of action patterns. However, before we proceed with action patterns discussion, we would like to summarize the assumptions used in this work:

Assumption 1. A process model collection is large enough to extract domain knowledge.

Assumption 2. An activity label signifies an action.

Assumption 3. There is a mechanism interpreting an activity label as an action.

\section{Action Patterns}

In general, a pattern is a concept that organizes knowledge related to "a problem which occurs over and over again in our environment, and then describes the core 
solution to that problem, in such a way that you can use this solution a million times over, without ever doing it the same way twice" [3]. While originally defined for architecture, this concept was adapted to software engineering in the 1990s (see 6]). In business process management, patterns have been defined, among others, for control flow [25], data flow [21], resources [22], and collaboration [15]. Also the MIT Process Handbook [16] can be related to the idea of describing a core solution to a recurring problem.

This section discusses the notion of actions patterns in order to meet the requirements for modeling support outlined above. First, Section 3.1 presents our formal framework for action patterns. Then, Section 3.2 defines co-occurrence action patterns. Finally, Section 3.3 specifies behavioral action patterns based on behavioral profiles.

\subsection{Formal Framework}

In order to formalize the concept of an action pattern we need to introduce a number of auxiliary concepts. First, we postulate $\Gamma$ to be the universal alphabet of labels. Based thereon, we define the notion of a process model enriched with labeling information.

Definition 1 (Process Model). A tuple $P M=(A, G, F, s, e, t, l)$ is a process model, where:

- $A$ is a finite nonempty set of activities;

- $G$ is a finite set of gateways;

- $A \cap G=\emptyset$ and $N=A \cup G$ is a finite set of nodes;

$-F \subseteq N \times N$ is the flow relation, such that $(N, F)$ is a connected graph;

$-s \in A$ is the only start activity, such that $\bullet s=\emptyset$, where $\bullet n=$ $\left\{n^{\prime} \in N \mid\left(n^{\prime}, n\right) \in F\right\}$ for node $n$;

$-e \in A$ is the only end activity, such that $e \bullet=\emptyset$, where $n \bullet=$ $\left\{n^{\prime} \in N \mid\left(n, n^{\prime}\right) \in F\right\}$ for node $n$;

- $t: G \mapsto\{$ and, xor, or $\}$ is a mapping that assigns type to each gateway;

$-l: A \mapsto \Gamma$ is a mapping assigning to each activity a label.

In the remainder, we do not formalize the execution semantics of a process model, but assume an interpretation of the model following on common execution semantics. Such semantics, in particular for the OR construct, has been presented in the existing work (see [17] as an example for EPCs).

To grasp the meaning of activities humans interpret their labels. In the context of this work interpretation of labels has great importance. Hence, we formalize it, introducing an alphabet of action terms $\mathcal{T}$ and a label interpretation function.

Definition 2 (Action Function). For a given process model $P M=$ $(A, G, F, s, e, t, l)$, the action function $v: \Gamma \mapsto \mathcal{T}$ derives an action from a label. As a shorthand notation, we introduce $v_{a}: A \mapsto \mathcal{T}$ for deriving an action from a label of an activity $a \in A$, i.e., $v_{a}(a)=v(l(a))$. We also use $V_{P M}=\bigcup_{a \in A}\left\{v_{a}(a)\right\}$ to denote the set of all actions of a process model. 
Revisiting the example containing the label send notification, application of the action function $v$ yields the action send. We also formalize the notion of a process model collection as follows.

Definition 3 (Process Model Collection). A tuple $C=(\mathcal{P} \mathcal{M}, V)$ is a process model collection, where:

$-\mathcal{P M}$ is a nonempty finite set of process models with elements $P M_{i}=$ $\left(A_{i}, G_{i}, F_{i}, s_{i}, e_{i}, t_{i}, l_{i}\right)$, where $i=1,2, \ldots,|\mathcal{P} \mathcal{M}|$;

- $V=\bigcup_{i=1,2, \ldots,|\mathcal{P M}|} V_{P M_{i}}$ is the set of all actions in the model collection.

It is natural to expect that in a large collection of process models one can observe sustainable relations between actions (action patterns). Recognition of action patterns resembles uncovering patterns in large data collections. The latter problem is in the focus of data mining. In particular we are interested in association rule learning - a well established technique for discovering relations between variables in large databases. An example of an association rule in a commerce domain is a statement that if customers buy coffee and milk, they usually buy sugar as well. Association rule learning enables discovery of such statements from the analysis of basket data in supermarkets. The initial idea of association rule learning was presented by Agrawal, Imielinski, and Swami in [1. More advanced algorithms were presented in [2].

Further, the generic formalism of association rule learning is adapted for both co-occurrence and behavioral action patterns. We introduce the set of items $\mathcal{I}$. Let us observe a collection of transactions $C$, where each transaction $T$ is a set of items, i.e., $T \subseteq \mathcal{I}$. Given a set of items $X \subseteq \mathcal{I}$, we say that transaction $T$ satisfies $X$, if $X \subseteq T$. An association rule in a collection $C$ is an implication of the form $X \Rightarrow Y$, where $X \cap Y=\emptyset$ and $X, Y \subset \mathcal{I}$.

Based thereon, two elementary notions can be defined, i.e., support and confidence. A set $X \subseteq \mathcal{I}$ has support $n$ in a collection $C$, if $n$ transactions satisfy set $X$. We denote the support for set $X$ with $\operatorname{supp}(X)$. Support can be related to statistical significance. In the context of action pattern retrieval we are interested in sets that have high support. Let us require the minimum level of support for sets to be minsup. Then $X$ is called a large set if $\operatorname{supp}(X) \geq \operatorname{minsup}$ (and a small set otherwise). An association rule $X \Rightarrow Y$ holds in transaction collection $C$ with confidence $c=\frac{\operatorname{supp}(X \cup Y)}{\operatorname{supp}(X)}$, if at least $c$ share of transactions satisfying $X$, satisfies $Y$ as well. The confidence for a rule $X \Rightarrow Y$ is denoted as $\operatorname{con} f(X \Rightarrow Y)$. A rule confidence reflects its strength. As in the case with support, we are interested in the rules with high confidence values. Hence, we introduce the minimal accepted level of confidence-minconf. Following [1, we claim that we are interested in the rules $X \Rightarrow Y$ for which $X \bigcup Y$ is large and the confidence is greater than user specified minconf.

\subsection{Co-occurrence Action Patterns}

The first class of action patterns is co-occurrence action patterns. Nomen est omen, these patterns capture sets of actions which often co-occur together in 
business processes, ignoring any ordering relations between these actions. In terms of association rules learning, we interpret actions as items and process models as transactions. Hence, a model collection is a collection of transactions. We say that a process model $P M=(A, G, F, s, e, t, l)$ satisfies an action set $X$, if $X \subseteq V_{P M}$. A co-occurrence action pattern is defined as an association rule on the domain of actions $V$ associated with values for minimal support and confidence.

\section{Definition 4 (Co-occurrence Action Pattern)}

$C A P=(R$, minsup, mincon $f)$ is a co-occurrence action pattern in process model collection $C=(\mathcal{P} \mathcal{M}, V)$, where:

$-R$ is an association rule $X \Rightarrow Y$, where $X, Y \subset V$;

- minsup is the value of the required minimal support;

- minconf is the value of the required minimal confidence.

From a user perspective such a pattern recommends the actions which are expected to appear in the process model given the current constellation of actions.

Mining of co-occurrence action patterns has two phases. In the first phase we seek for association rules $X \Rightarrow Y$, such that $X \bigcup Y$ is a large set. In the second phase the mined large sets are used for derivation of patterns - rules that have a high confidence level.

A search for large sets is a computationally intensive task. In this paper we set our choice on Apriori algorithm, since it is efficient and simple [2]. In terms of large action sets this algorithm works as follows. As the input the algorithm takes the process model collection $C=(\mathcal{P} \mathcal{M}, V)$ and the minimal support value minsup. For every action $v \in V$, a one element action set is constructed, $\{v\}$. Then, for each action set, the algorithm checks its support. If the support is not less than minsup, the set is large. The derived 1-large sets are used as the input for the next step. In the $k$-th step the algorithm constructs sets of size $k$ from $k-1$ large sets and checks if they are large. The algorithm terminates, once all the large sets are found. Table 1 illustrates the first (see Table [1](b)] and the second steps (see Table[1](c)] of Apriori work for the model collection captured in Table 1/(a) given minsup $=5$.

After large sets have been retrieved, the second phase explores each large set for rules with high confidence level. A rule $A \Rightarrow B$ is defined by two sets: antecedent $(A)$ and consequent $(B)$. We consider all possible partitions of a large set into two sets, one of them to become an antecedent and the other - a consequent. For each partitioning we check, if it results in a rule with a confidence level greater than minsup.

\subsection{Behavioral Action Patterns}

Co-occurrence action patterns do not provide information about how the missing actions have to be introduced into the process model. As the next step, we consider action patterns that are enriched with information on relations between actions. First, we present preliminaries on behavioral relations and afterwards introduce the notion of a behavioral action pattern. 
Table 1. Derivation of large action sets in a process model collection given minsup $=5$

(a) Process model collection

\begin{tabular}{ll}
\hline Model & Actions \\
\hline A & allocate analyze calculate collect evaluate settle summarize \\
B & $\begin{array}{l}\text { allocate analyze asses calculate distribute entry evaluate reconcile repost } \\
\text { settle split }\end{array}$ \\
C & allocate analyze calculate cost settle \\
D & allocate analyze calculate evaluate settle \\
E & allocate analyze collect calculate distribute evaluate settle summarize \\
F & allocate budget calculate copy define evaluate plan reconcile settle split \\
& transfer \\
G & allocate budget calculate copy cost define plan reconcile settle split transfer \\
\hline
\end{tabular}

(b) Large action sets of size 1

\begin{tabular}{ll}
\hline Set & Support \\
\hline allocate\} & 7 \\
\{analyze\} & 5 \\
\{calculate & 7 \\
\{evaluate\} & 5 \\
settle\} & 7 \\
\hline
\end{tabular}

(c) Large action sets of size 2

\begin{tabular}{ll}
\hline Set & Support \\
\hline allocate, analyze & 5 \\
\{allocate, calculate & 7 \\
\{allocate, evaluate & 5 \\
allocate, settle\} & 7 \\
analyze, calculate & 5 \\
analyze, settle\} & 5 \\
\{calculate, evaluate & 5 \\
\{calculate, settle\} & 7 \\
\{evaluate, settle\} & 5 \\
\hline
\end{tabular}

Behavioral Relations. In order to capture behavioral aspects of a process on the level of pairs of activities, we apply the notion of behavioral profiles [27. Although Definition 1 does not specify execution semantics, we impose syntactical requirements for the definition of all complete traces of a process model to define its behavioral profile. That is, the (potentially unbounded) set of complete process traces $\mathcal{T}_{P M}$ for a process model $P M=(A, G, F, s, e, t, l)$ is a set of lists of the form $s \cdot A^{*} \cdot e$, such that a list entry contains the execution order of activities. Further on, we use $a \in \sigma$ with $\sigma \in \mathcal{T}_{P M}$ to denote that an activity $a \in A$ is a part of a complete process trace. The behavioral profile is grounded on the notion of weak order. Two activities of a process model are in weak order, if there exists a trace in which one node occurs after the other.

Definition 5 (Weak Order Relation). Let $P M=(A, G, F, s, e, t, l)$ be a process model, and $\mathcal{T}_{P M}$-its set of traces. The weak order relation $\succ_{P M} \subseteq$ $(A \times A)$ contains all pairs $(x, y)$, such that there is a trace $\sigma=n_{1}, \ldots, n_{m}$ in $\mathcal{T}_{P M}$ with $j \in\{1, \ldots, m-1\}$ and $j<k \leq m$ for which holds $n_{j}=x$ and $n_{k}=y$.

Depending on how two activities of a process model are related by weak order, we define three relations forming the behavioral profile. 
Definition 6 (Behavioral Profile). Let $P M=(A, G, F, s, e, t, l)$ be a process model. A pair $(x, y) \in(A \times A)$ is in one of the following relations:

- strict order relation $\rightsquigarrow P M$, if $x \succ_{P M} y$ and $y \nsucc_{P M} x$;

- exclusiveness relation $+_{P M}$, if $x \nsucc_{P M} y$ and $y \nsucc_{P M} x$;

- observation concurrency relation $\|_{P M}$, if $x \succ_{P M} y$ and $y \succ_{P M} x$.

The set of all three relations is the behavioral profile of $P M$.

We illustrate the behavioral profile by means of the model in Fig. 2, For instance, (Template allocation) $\rightsquigarrow$ (Overhead calculation) holds as there exists no trace, such that the latter function occurs before the former. With $\rightsquigarrow-1$ as the inverse relation for $\rightsquigarrow P M$, (Revaluation completed) $\rightsquigarrow \frac{-1}{P M}$ (Template allocation) also holds. It is worth to mention that $\rightsquigarrow P M, \rightsquigarrow \frac{-1}{P M},+_{P M}$, and $\|_{P M}$ partition the Cartesian product of activities $A \times A$ for a process model $P M=(A, G, F, s, e, t, l)$.

\section{The Concept of Behavioral Action Pat-}

terns. We introduce behavioral action patterns as a mechanism enabling suggestions on how the missing actions should be introduced in an existing process model. Such patterns provide more information to the user than co-occurrence action patterns. However, we perceive behavioral patterns not as a mechanism replacing co-occurrence patterns, but rather as a complimentary mechanism: while co-occurrence action patterns suggest which actions are missing, behavioral action patterns hints on action relations. Assume a user designs a process model containing actions allocate and calculate; co-occurrence action pattern $\{$ allocate, calculate $\} \Rightarrow\{$ settle $\}$ is available (see Fig. 2). This pattern suggests to add action settle in the process model. Then, we can look up a suitable behavioral action pattern describing relations between these three actions. Behavioral action pattern $\{$ allocate $\rightsquigarrow$ calculate $\} \Rightarrow$ allocate $\rightsquigarrow$ settle, calculate $\rightsquigarrow$ settle $\}$ provides a desired

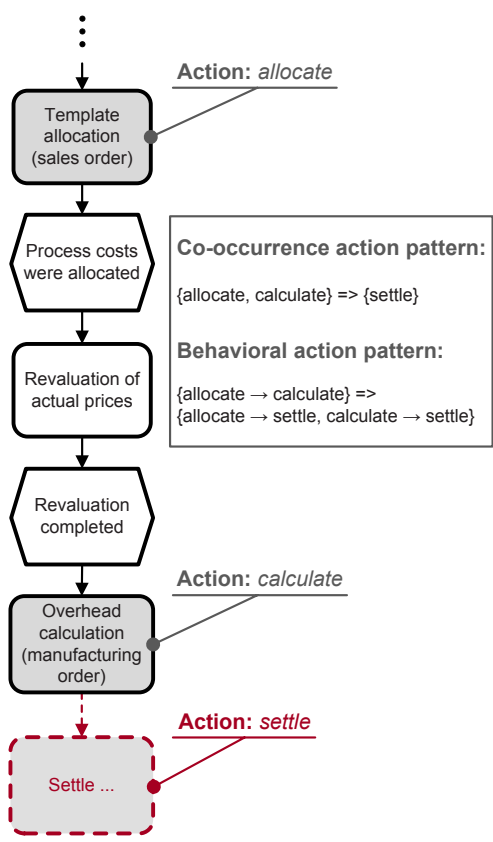

Fig. 2. Exemplary suggestion based on action patterns recommendation.

To formalize the concept of relations between actions, we propose to adapt the behavioral relations between activities introduced earlier. We say that actions $v_{1}$ and $v_{2}$ are in relation $R$ in a process model $P M=(A, G, F, s, e, t, l)$, if there are two activities $a, b \in A$, such that $(a, b) \in R \wedge v_{a}(a)=v_{1} \wedge v_{a}(b)=v_{2}$. Within one process model a pair of actions $\left(v_{1}, v_{2}\right)$ may be in more than one relation. This holds if there are several activities that signify action $v_{1}$, or action $v_{2}$, or both actions. 
Definition 7 (Behavioral Action Pattern). BAP $=(R$, minsup, minconf $)$ is a behavioral action pattern in process model collection $C=(\mathcal{P} \mathcal{M}, V)$, where:

$-R$ is a rule $X \Rightarrow Y$, where $X, Y \subset V \times\left\{\rightsquigarrow, \rightsquigarrow^{-1},+, \|\right\} \times V$, i.e., $X$ and $Y$ constitute of pairs of actions for which behavioral relations are specified;

- minsup is the value of the required minimal support;

- minconf is the value of the required minimal confidence.

Mining of behavioral action patterns resembles the approach introduced for cooccurrence action patterns. In the first phase we seek for large action sets. In the second phase we inspect the relations between the actions of each large set. In terms of association rules derivation, action relations are treated as items, while large action sets are interpreted as collections. Provided minsup and minconf values, we can derive behavioral action patterns.

\section{Evaluation Based on the SAP Reference Model}

To validate the proposed concepts and algorithms, we have conducted an experiment. The goals of the experiment were: 1) to check if it is possible to derive action patterns from a collection of process models and 2) to learn which support and confidence values are encountered in practice. The experiment consists of two parts: in the first part co-occurrence action patterns have been studied, in the second-behavioral action patterns.

The experiment studies the SAP Reference Model [9], a process model collection that has been used in several works on process model analysis [17. The collection captures business processes that are supported by the SAP R/3 software in its version from the year 2000. It is organized in 29 functional branches of an enterprise, like sales or accounting, that are covered by the SAP software. The SAP Reference Model includes 604 Event-driven Process Chains (EPCs). All of these models have been considered in the first part of experiment for deriving co-occurrence patterns. In the second part, inspecting behavioral action patterns, the number of models was 421 . The decrease in the model number is due to the exclusion of models with ambiguous instantiation semantics (see [5]) or behavioral anomalies (see [17]). At this stage we derived actions from activity labels manually. We foresee that this step can be automated in the future and are currently investigating techniques enabling the automation.

In the first part of the experiment, we have derived co-occurrence action patterns. The first question to be answered is which values of support and confidence indicate relevant patterns. While higher values indicate that the pattern is more reliable, we aim to understand which values can be expected. In the SAP Reference Model the support value for all action sets is under 10, which is quite low given the fact that some actions appear several hundred times [18]. As the minimally acceptable confidence level is hard to predict, we conducted a set of experiments varying the level of support from 2 to 9 and the level of confidence from 0.5 to 0.95 . Table 2 summarizes the results of these experiments. It shows that there is almost half a million patterns with support 2, 17 patterns with support of 9 , and not a single pattern has support 10 . To illustrate how the derived 
Table 2. Dependency of co-occurrence pattern number in the SAP Reference Model on minsup and minconf values

\begin{tabular}{|c|c|c|c|c|c|c|c|c|c|}
\hline minconf & insup & 2 & 3 & 4 & 5 & 6 & 7 & 8 & 9 \\
\hline & 0.50 & 522396 & 7395 & 2353 & 680 & 563 & 41 & 29 & 17 \\
\hline & 0.55 & 511373 & 6979 & 2247 & 665 & 550 & 34 & 23 & 13 \\
\hline & 0.60 & 510517 & 6123 & 2089 & 610 & 504 & 33 & 22 & 12 \\
\hline & 0.65 & 510498 & 6104 & 2070 & 591 & 497 & 26 & 16 & 9 \\
\hline & 0.70 & 484061 & 5569 & 1535 & 563 & 469 & 20 & 12 & 6 \\
\hline & 0.75 & 483415 & 4923 & 1477 & 505 & 421 & 19 & 11 & 6 \\
\hline & 0.80 & 483176 & 4684 & 1238 & 501 & 417 & 15 & 10 & 5 \\
\hline & 0.85 & 483135 & 4643 & 1197 & 460 & 417 & 15 & 10 & 5 \\
\hline & 0.90 & 483095 & 4603 & 1157 & 420 & 377 & 7 & 3 & 2 \\
\hline & 0.95 & 483093 & 4601 & 1155 & 418 & 375 & 5 & 1 & 0 \\
\hline
\end{tabular}

Table 3. Derived behavioral profiles for action set \{allocate, calculate, settle\}

\begin{tabular}{ccccc}
\hline Model & Action pair & (allocate, calculate) & (allocate, settle) & (calculate, settle) \\
\hline & A & $\rightsquigarrow$ & $\rightsquigarrow$ & $\rightsquigarrow$ \\
B & $\rightsquigarrow$ & $\rightsquigarrow$ & $\rightsquigarrow$ \\
C & $\rightsquigarrow$ & $\rightsquigarrow$ & $\rightsquigarrow$ \\
D & $\rightsquigarrow$ & $\rightsquigarrow$ & \\
\hline
\end{tabular}

patterns look like, we zoom into one cell of the table and list the patterns with minsup $=7$ and minconf $=0.95$ :

$-\{$ pick $\} \Rightarrow\{$ process $\}$

$-\{$ level $\} \Rightarrow\{$ evaluate $\}$

$-\{$ permit $\} \Rightarrow\{$ process $\}$

$-\{$ archive, enter $\} \Rightarrow\{$ process $\}$

- $\{$ allocate, calculate $\} \Rightarrow\{$ settle $\}$

The results show that for the studied model collection the maximum support value is small. On the one hand, this is caused by unsystematic usage of labels: often the derived actions are semantically close, but are treated as different actions. On the other hand, this fact can be explained by the heterogeneity of process models. The presence of process variants in the collection leads to the fact that some action patterns, especially of size 5-7, identify these variants in the model set.

Behavioral action patterns originate from the inspection of behavioral constraints between actions in large action sets. Hence, derivation of behavioral patterns is possible only after minsup for action sets is given. In the experiment we considered those process models from the SAP Reference Model that can be mapped to free-choice Petri nets. Table 3 provides an example of relations for actions allocate, calculate, settle. Table $4(\mathrm{a})$ shows the number of patterns that 
Table 4. Dependency of behavioral action patterns number for 2 action sets on minsup and minconf values

(a) Action set \{allocate, calculate, settle\} (b) Action set \{analyze, allocate, settle\}

\begin{tabular}{|c|c|c|c|c|c|c|c|c|c|c|c|c|}
\hline minconf & minsup & 2 & 3 & 4 & 5 & minconf & & 2 & 3 & 4 & & 6 \\
\hline & 0.50 & 12 & 12 & 12 & 0 & & 0.50 & 170 & 12 & 4 & 2 & 0 \\
\hline & 0.55 & 12 & 12 & 12 & 0 & & 0.55 & 161 & 12 & 4 & 2 & 0 \\
\hline & 0.60 & 12 & 12 & 12 & 0 & & 0.60 & 157 & 8 & 3 & 2 & 0 \\
\hline & 0.65 & 12 & 12 & 12 & 0 & & 0.65 & 157 & 8 & 3 & 2 & 0 \\
\hline & 0.70 & 12 & 12 & 12 & 0 & & 0.70 & 130 & 8 & 3 & 2 & 0 \\
\hline & 0.75 & 12 & 12 & 12 & 0 & & 0.75 & 129 & 7 & 2 & 1 & 0 \\
\hline & 0.80 & 12 & 12 & 12 & 0 & & 0.80 & 129 & 7 & 2 & 1 & 0 \\
\hline & 0.85 & 12 & 12 & 12 & 0 & & 0.85 & 129 & 7 & 2 & 1 & 0 \\
\hline & 0.90 & 12 & 12 & 12 & 0 & & 0.90 & 129 & 7 & 2 & 1 & 0 \\
\hline & 0.95 & 12 & 12 & 12 & 0 & & 0.95 & 129 & 7 & 2 & 1 & 0 \\
\hline
\end{tabular}

can be derived for this set depending on the minsup and minconf values for relations. A more vivid example is the action set $\{$ analyze, allocate, settle $\}$, for which the number of behavioral patterns varies greatly (see Table 4 (b)]. A concrete example of a behavioral action pattern which can be derived from Table 3 is $\{$ allocate $\rightsquigarrow$ calculate $\} \Rightarrow$ allocate $\rightsquigarrow$ settle, calculate $\rightsquigarrow$ settle $\}$. This pattern prescribes that the three actions are sequentially constrained. They should appear in the process model such that first it is allocated, then calculated, and finally settled, which is a standard sequence of activities for financial assets.

\section{Related Work}

Our work can be related to different contributions to business process modeling. We focus on the three areas, i.e., patterns for business processes, intelligent modeling support, and research on activity labels.

There is a wide variety of patterns proposed for business processes and business process modeling. On the technical level, the workflow pattern initiative has identified various patterns for the specification of control flow [25], data flow 21], and resources 22] in workflow management systems. On a more conceptual level, Lonchamp proposed a set of collaboration patterns defining abstract building blocks for recurrent situations [15]. Tran et al. formalize process patterns using UML concepts 24. Most closely related to our work is the research by Thom et al. 23]. The authors identify so-called activity patterns that specify eight different types of micro workflows, like approval or decision. Further, in [14] the authors describe a method for patterns derivation. While [1423] operates directly with activities, we use the concept of actions. As in real world models activities with different labels often signify the same action, usage of actions facilitates pattern derivation. Next, instead of direct analysis of a model 
graph structure, we rely on the concept of behavioral profiles. As relations captured by behavioral profiles are weaker than those defined by process models, 14 discovers only a subclass of behavioral action patterns.

The potential of improving business process modeling using intelligent support and recommendations has been recognized only recently. Hornung et al. define a concept to provide recommendations to the modeler based on search techniques 8]. The idea is to find similar models in the process repository and propose them as extensions to a process being currently modeled. This idea is in line with our approach, but requires a match not only in terms of actions, but also business objects and other textual content. We deem our approach to be more flexible and applicable across different modeling contexts. Further experiments are needed to check comparative strengths and weaknesses. A different stream of research investigates how far social software and Web 2.0 applications can provide recommendations to the modeler. Koschmider et al. propose a solution that enables collaborative modeling and user recommendations [11]2. In contrast to our work, the approach builds on behavior and suggestions of other modelers. Control flow correctness issues are addressed in [13, where the authors offer continuous verification of process models during modeling. In [10] the authors study how cooperative modeling is supported by fragment-driven modeling approach. However, this paper primary focuses on describing the infrastructure for cooperative modeling, but not on the derivation of fragments (or action patterns). Gschwind et al. employ control flow patterns to accelerate business process modeling and minimize the number of modeling errors [7. The authors develop a suggestion mechanism considering structural patterns and the model structure at hand.

Recent contributions identify a textual analysis of activity labels as an important step to improve the pragmatic quality of process models. For instance, different labeling schemata and their impact on model understanding have been analyzed in [19. Textual labels are also used for matching and comparing process models [8 26]. Recent works by Becker et al. reuse parsing techniques from computer linguistics to efficiently identify the various parts of an activity label [4]. While we have derived the actions manually for our experiment reported in this paper, we are currently working on automating this step by using the approach taken by Becker et al.

\section{Conclusion}

In this paper we have addressed the challenge of assisting the designer in modeling a process. We defined the concept of action patterns, capturing co-occurrences of actions in existing process model collections. Our contribution is an approach based on association rules mining that identifies sets of actions that likely imply further actions. In this way, action patterns can be used to suggest additional activities to the modeler. Furthermore, we utilize behavioral profiles to capture behavioral relations between co-occurring actions. Therefore, we also provide information on how the additional action should be included in the process model. Our approach has been validated using the SAP Reference Model. 
We assume that a mapping of an activity label to an action is given. However, derivation of actions from labels is a challenging research topic and is in the focus of our future work. Further, we analyze activity labels to derive actions only. We do not consider business objects referenced in the labels of model elements as well. While this might be regarded as a limitation, we made this design choice to identify recurring patterns holding for different business objects. Taking business objects into account offers several advantages, including object life cycle mining. A mined object life cycle is a helpful tool as it facilitates advanced modeling support. Another potential direction of the future work involves synonym recognition. Usage of thesauri, like WordNet, would allow to cluster actions that are closely related and gain stronger support for related patterns. In this context, one might also consider action hierarchies like the one developed for the MIT Process Handbook.

As process model collections are often incremented with new models, methods for action patterns derivation have to be efficient and adaptive. While in this paper derivation of action patterns relies on Apriori algorithm, there is a potential to improve the performance by substituting Apriori algorithm with a more efficient one. In addition, efficient strategies for adjusting the set of action patterns after creation of new process models have to be evaluated. Obviously, support and confidence values of existing patterns might easily be adapted. However, the detection of additional patterns, which have been ignored due to low support and confidence values before the model collection has been incremented, remains a serious issue. All these directions are rather unexplored for process models, and are on our future research agenda.

\section{References}

1. Agrawal, R., Imielinski, T., Swami, A.N.: Mining Association Rules between Sets of Items in Large Databases. In: COMAD, Washington, D.C, pp. 207-216 (1993)

2. Agrawal, R., Srikant, R.: Fast Algorithms for Mining Association Rules in Large Databases. In: VLDB, pp. 487-499. Morgan Kaufmann Publishers Inc., San Francisco (1994)

3. Alexander, C., Ishikawa, S., Silverstein, M.: A Pattern Language: Towns, Buildings, Construction. Oxford University Press, New York (1977)

4. Becker, J., Delfmann, P., Herwig, S., Lis, L., Stein, A.: Towards Increased Comparability of Conceptual Models - Enforcing Naming Conventions through Domain Thesauri and Linguistic Grammars. In: ECIS (June 2009)

5. Decker, G., Mendling, J.: Instantiation Semantics for Process Models. In: Dumas, M., Reichert, M., Shan, M.-C. (eds.) BPM 2008. LNCS, vol. 5240, pp. 164-179. Springer, Heidelberg (2008)

6. Gamma, E., Helm, R., Johnson, R., Vlissides, J.: Design Patterns. Addison-Wesley, Boston (1995)

7. Gschwind, T., Koehler, J., Wong, J.: Applying Patterns during Business Process Modeling. In: Dumas, M., Reichert, M., Shan, M.-C. (eds.) BPM 2008. LNCS, vol. 5240, pp. 4-19. Springer, Heidelberg (2008)

8. Hornung, T., Koschmider, A., Lausen, G.: Recommendation Based Process Modeling Support: Method and User Experience. In: Li, Q., Spaccapietra, S., Yu, E., Olivé, A. (eds.) ER 2008. LNCS, vol. 5231, pp. 265-278. Springer, Heidelberg (2008) 
9. Keller, G., Teufel, T.: SAP $(\mathrm{R}) \mathrm{R} / 3$ Process Oriented Implementation: Iterative Process Prototyping. Addison-Wesley, Reading (1998)

10. Kim, K.-H., Won, J.-K., Kim, C.-M.: A Fragment-Driven Process Modeling Methodology. In: Gervasi, O., Gavrilova, M.L., Kumar, V., Laganá, A., Lee, H.P., Mun, Y., Taniar, D., Tan, C.J.K. (eds.) ICCSA 2005. LNCS, vol. 3482, pp. 817-826. Springer, Heidelberg (2005)

11. Koschmider, A., Song, M., Reijers, H.A.: Social Software for Modeling Business Processes. In: Ardagna, D., et al. (eds.) BPM 2008 Workshops. LNBIP, vol. 17, pp. 642-653. Springer, Heidelberg (2009)

12. Koschmider, A., Song, M., Reijers, H.A.: Advanced Social Features in a Recommendation System for Process Modeling. In: Abramowicz, W. (ed.) BIS 2009. LNBIP, vol. 21, pp. 109-120. Springer, Heidelberg (2009)

13. Kühne, S., Kern, H., Gruhn, V., Laue, R.: Business Process Modelling with Continuous Validation. In: MDE4BPM, September 2008, pp. 37-48 (2008)

14. Lau, J.M., Iochpe, C., Thom, L., Reichert, M.: Discovery and Analysis of Activity Pattern Cooccurrences in Business Process Models. In: ICEIS, pp. 83-88. Springer, Heidelberg (2009)

15. Lonchamp, J.: Process Model Patterns for Collaborative Work. In: Telecoop (1998)

16. Malone, T.W., Crowston, K., Herman, G.A.: Organizing Business Knowledge: The MIT Process Handbook, 1st edn. The MIT Press, Cambridge (2003)

17. Mendling, J.: Metrics for Process Models: Empirical Foundations of Verification, Error Prediction, and Guidelines for Correctness. In: Mendling, J. (ed.) Metrics for Process Models. LNBIP, vol. 6, pp. 1-15. Springer, Heidelberg (2008)

18. Mendling, J., Recker, J.: Towards Systematic Usage of Labels and Icons in Business Process Models. In: EMMSAD, June 2008, vol. 337, pp. 1-13. CEUR Workshop Proceedings (2008)

19. Mendling, J., Reijers, H.A., Recker, J.: Activity Labeling in Process Modeling: Empirical Insights and Recommendations. Information Systems (to appear, 2009)

20. Rosemann, M.: Potential Pitfalls of Process Modeling: Part A. Business Process Management Journal 12(2), 249-254 (2006)

21. Russell, N., ter Hofstede, A.H.M., Edmond, D., van der Aalst, W.M.P.: Workflow Data Patterns. Technical Report FIT-TR-2004-01, QUT (2004)

22. Russell, N., van der Aalst, W.M.P., ter Hofstede, A.H.M., Edmond, D.: Workflow Resource Patterns. Technical Report WP 126, Eindhoven University of Technology (2004)

23. Thom, L.H., Reichert, M., Chiao, C.M., Iochpe, C., Hess, G.N.: Inventing Less, Reusing More, and Adding Intelligence to Business Process Modeling. In: Bhowmick, S.S., Küng, J., Wagner, R. (eds.) DEXA 2008. LNCS, vol. 5181, pp. 837-850. Springer, Heidelberg (2008)

24. Tran, H.N., Coulette, B., Dong, B.T.: Broadening the Use of Process Patterns for Modeling Processes. In: SEKE, July 2007, pp. 57-62. Knowledge Systems Institute Graduate School (2007)

25. van der Aalst, W.M.P., ter Hofstede, A.H.M., Kiepuszewski, B., Barros, A.P.: Workflow Patterns. Distrib. Parallel Databases 14(1), 5-51 (2003)

26. van Dongen, B., Dijkman, R., Mendling, J.: Measuring Similarity between Business Process Models. In: Bellahsène, Z., Léonard, M. (eds.) CAiSE 2008. LNCS, vol. 5074, pp. 450-464. Springer, Heidelberg (2008)

27. Weidlich, M., Mendling, J., Weske, M.: Computation of Behavioural Profiles of Process Models. Technical report, HPI (June 2009), http://bpt.hpi.uni-potsdam.de/pub/Public/MatthiasWeidlich/ behavioural_profiles_report.pdf 